familiarize themselves with the ARS units at their seats. Five hundred ARS units, provided by Merck US Human Health, were available for each session. Each unit was a hand-held device with a numbered keypad on its surface. Audience members were instructed to enter their responses by pressing the key with the corresponding answer number. They were given 15 to 25 seconds to answer each question after it was read by the moderator; total time depended on the number of possible answer choices. The last answer entered during the allotted time was captured by the ARS system and tallied. The final number of respondents and percentages for each answer option subsequently were displayed for audience members and the session presenters.

The same five demographic questions were asked at the beginning of each session (Table 2). This was followed by further discussion and specific topical questions. The first six sessions were approximately 45 minutes in length, and the seventh session was 90 minutes. For most sessions, a topical question was asked, and then discussants commented on the results of the audience tally. The sessions on control of vancomycin-resistant Enterococcus and use of vancomycin prophylaxis involved 10-minute presentations by each discussant before topical questions were asked. The session on post-hepatitis $C$ virus exposure management was conducted using a case-study model and involved additional audience participation.

Tallied data were used in combination with audiotape recordings of the sessions to review and analyze audience and discussant responses.

\title{
Legionnaires' Disease From Potting Soil
}

Gina Pugliese, RN, MS Martin S. Favero, PhD

Since legionnaires' disease (LD) was first reported in 1976, outbreaks have been associated with airborne transmission of Legionella bacteria through cooling towers, showers, and other aerosolizing devices. However, most LD cases are sporadic, and the source and mode of infection in many cases are unknown. Infections with one species, Legionella longbeachae, have been associated with gardening and use of potting soil in Australia and Japan. A recent report from the $\mathrm{CDC}$ summarizes the findings of $\mathrm{LD}$ investigations of three cases in California, Oregon, and Washington that suggest that transmission from potting soil has occurred for the first time in the United States, and that active surveillance and case finding are warranted to explore this association. In two of the three cases, the patient had been potting plants during the 10 days before symptoms began, and $L$ longbeachae was isolated from the patient sputum or bronchial wash and a potting soil sample. In the third case, the patient was found to have $L$ longbeachae in her sputum, but no samples were available from the home.

Legionella pneumophila serogroup 1 accounts for most legionellosis cases in the United States; L longbeachae is reported less frequently. During 1990 to 1999,37 cases of $L$ longbeachae were reported to the CDC's Legionella reporting system. It is likely that legionellosis is underreported to the CDC because of failure to obtain the appropriate diagnostic tests in cases of pneumonia of unknown etiology, difficulty of growing cultures of Legionella from clinical specimens, and because legionellosis is not reportable in all states.

Soil surveys for Legionella have not been conducted in the United States. In a soil survey in Australia, 33 (73\%) of 45 potting soil samples tested positive for
Legionella; 26 (79\%) of the 33 contained $L$ longbeachae. Nineteen (100\%) soil samples in Europe and the United Kingdom were negative for L longbeachae. A survey of 17 soil samples in Japan in 1998 yielded 31 different strains of Legionella; 8 of the 17 samples $(47 \%)$ contained $L$ longbeachae.

The CDC asks that cases of $L$ longbeachae infection that have occurred during the previous 12 months be reported to the CDC's National Center for Infectious Diseases, Division of Bacterial and Mycotic Diseases, Respiratory Diseases Branch, telephone 404-639-2215. For state and local use, a case-report form may be obtained from http://www.cdc.gov/ncidod/dbmd/ diseaseinfo/legionellosis_a.htm, and faxed to 404-639-3970.

FROM: CDC. Legionnaires' disease associated with potting soil, California, Oregon, Washington-May-June 2000. MMWR 2000;49:777-778. 